\title{
Foetal hepatorenal toxicity and intrauterine growth retardation in pregnant Wistar rats treated with artemether
}

\author{
Idorenyin Udo Umoh ${ }^{1}$, Etukudoh Okon Jimmy ${ }^{2}$ \\ ${ }^{1}$ Department of Anatomy, Faculty of Basic Medical Sciences, University of Uyo, Uyo, Akwa Ibom State, Nigeria \\ ${ }^{2}$ Department of Physiology, Faculty of Basic Medical Sciences, University of Uyo, Uyo, Akwa Ibom State, Nigeria
}

\begin{abstract}
Objectives: Foetal hepatorenal toxicity and foetal morphology were evaluated following artemether administration to pregnant Wistar albino rats.

Methods: Twenty pregnant Wistar rats weighing between 180-200 g were divided into four groups ( $\mathrm{n}=5$, each) with Group 1 serving as the control. Groups 2, 3 and 4 received $1.1 \mathrm{mg}, 2.2 \mathrm{mg}$ and $3.3 \mathrm{mg}$ per kilogram body weight artemether, respectively orally, twice daily for three days on day 7,8 and 9 of pregnancy. The animals were sacrificed on day 20 of pregnancy and foetuses were harvested and evaluated for morphological changes. Foetal body weight and crown-rump length (CRL) were measured; alpha-fetoprotein (AFP) was assayed using amniotic fluid, and foetal kidney and liver were evaluated histologically for toxicity.

Results: There was a significant decrease in body weight, CRL and AFP of the treated groups compared to the control. The foetal liver of the treated groups revealed distorted cytoarchitecture, marked hepatocyte inflammation and hepatic necrosis. The foetal kidney of artemether-treated groups also showed disorganised renal structure, atrophic and degenerated glomeruli with acute
\end{abstract} tubular necrosis.

Conclusion: Artemether administration to pregnant albino rats causes intrauterine growth retardation or stunted growth, as well as foetal hepatorenal toxicity.

Keywords: alpha-fetoprotein; artemether; foetal hepatorenal toxicity; pregnancy

Anatomy 2018;12(1):1-6 ()2018 Turkish Society of Anatomy and Clinical Anatomy (TSACA)

\section{Introduction}

Malaria is one of the commonest tropical diseases which is a major source of concern to the developing world in terms of morbidity and mortality in children and pregnant women, especially with the emergence of the resistance of the Plasmodium parasite ${ }^{[1]}$ to common, affordable and readily available anti-malarial drugs. In 1998, about 373 million people were infected with malaria. In 2008, about 247 million cases of malaria were reported and nearly one million deaths were recorded annually among children living in Africa. ${ }^{[2]}$ The World Malaria Report of 2014 showed that children under the age of 5 years are at high risk of malaria and they account for about $78 \%$ of deaths resulting from malaria. ${ }^{[1]}$ Recent occurrence of malaria is estimated at 198 million (uncertainty range 124 million to 283 million) cases with estimated 367,000 to 755,000 deaths annually,
90\% of them in Africa. ${ }^{[1]}$ These statistics have shown a decrease in both the occurrence and mortality of malaria over the years, but the figures are still alarming and a cause for major concern.

Plasmodium falciparum is the most virulent of all Plasmodium species that cause malaria and is known to have the highest morbidity and mortality rate..$^{[3]}$ Malaria is transmitted from one infected person to another through a bite of an infected female Anopheles mosquito as vector. However, the intensity of transmission is dependent on factors related to the parasites, the vector, human host and the environment. Malaria is however a preventable and curable disease. Pregnant women are also very susceptible to malaria infection hence it constitutes a threat to both women and the foetuses. 
The prevalence of Plasmodium falciparum resistance to antimalarials especially in endemic regions further narrows the spectrum of antimalarial drugs available for use during pregnancy. The World Health Organization (WHO) has recommended the use of artemisinin-based combination therapy (ACT) for treatment of malaria in second and third trimesters of pregnancy. ${ }^{[2]}$ Readily available, WHO-recommended ACTs include artemether-lumefantrine, artesunate-amodiaquine, artesunate-mefloquine, dihydroartemisinin-piperaquine and artesunate-sulfadoxinepyrimethamine. These drugs have been known to be very effective in the treatment and management of malaria, and several studies have been carried out to evaluate the effect of these therapies on the biological system. ${ }^{[4]}$

Studies have focused on the effect of artemisinins during pregnancy in experimental animals. ${ }^{[9]}$ The effect of artemether during different phases of pregnancy has been investigated in blastogenesis, organogenesis and foetal period. ${ }^{[10]}$ At doses of 3.5 and $7 \mathrm{mg} / \mathrm{kg} / \mathrm{bw}$, artemether was observed to be lethal to embryos during organogenesis, and foetal growth retardation without incidence of malfunction was also observed. Furthermore, severe embryotoxicity has been reported for artemisinins, especially injectable artesunate at low doses in rodent, but embryotoxicity has not been convincingly observed in humans in clinical trials. ${ }^{[11]}$ Embryo lethality and foetal cardiovascular as well as skeletal abnormalities have been observed for all derivatives of artemisinin at high doses. ${ }^{[12]}$ Longo et al. ${ }^{[13]}$ studied the effect of dihydroartemisinin on rat embryos and suggested that embryotoxicity of artemisinins may be due to oxidative stress induced by the drug.

Alpha-fetoprotein (AFP) is measured in pregnant women using maternal blood or amniotic fluid as a screening test for a subset of developmental abnormalities. AFP is particularly increased in open neural tube defect (NTD) and decreased in Down syndrome. It has also been used as a biomarker to detect tumours. ${ }^{[14]}$ Foetal crown-rump length and body weight give an indication of foetal growth.

This study was designed to evaluate the foetal hepatorenal toxicity and intrauterine growth retardation following administration of artemether to pregnant Wistar albino rats.

\section{Materials and Methods}

Twenty pregnant female Wistar albino rats weighing between 180-200 $\mathrm{g}$ were used for the study. The rats were obtained from the animal house of the Department of Pharmacology, University of Calabar, Calabar, Nigeria and acclimatized for two weeks at the Animal House of Faculty of Basic Medical Sciences, University of Uyo, Nigeria. The approval for the study and the use of animals was obtained from the Faculty Animal Care and Use Committee of the Faculty of Basic Medical Sciences of University of Uyo, Uyo, Nigeria. Rules and regulations of Institute for Animal Ethical Committee (IAEC) were strictly followed and ethical standards from 1964 declaration of Helsinki were followed. The rats were fed with pellets and clean drinking water ad libitum. The rats were randomly divided into 4 groups of 5 per group. Female rats in proestrus phase of their cycle were transferred into a cage overnight with matured sexually active male Wistar albino rats. The vaginal aspirates were taken and smear done following overnight mating to demonstrate the presence of spermatozoa and this was taken to signify day zero of pregnancy. Group 1 served as control while Groups 2-4 received $1.1 \mathrm{mg}, 2.2 \mathrm{mg}, 3.3 \mathrm{mg}$ of artemether per kilogram of body weight (bw) daily for five days between day 7 and day 11 of pregnancy.

Artemether manufactured by Greenlife Pharmaceuticals, Lagos, Nigeria was purchased from a registered pharmaceutical store in Uyo, Akwa Ibom State, Nigeria. Stock solution was prepared from the tablets and aliquots administered to the animals using cannula bypassing the oesophagus and delivered into the stomach based on the body weight.

The animals were sacrificed on the 20th day of the pregnancy under chloroform anaesthesia. The uterus was examined for the number of implantation sites, number of resorption sites and viable foetuses. The foetuses were delivered by uterotomy and examined for abnormal external features. Crown-rump length of each foetus was measured and the foetuses were weighed. The foetal liver and kidney were harvested and fixed in 10\% formalin for histological studies using haemotoxylin and eosin techniques. Amniotic fluid was also collected for analysis of AFP using the alpha fetoprotein kits.

The preserved tissues were dehydrated with ascending grades of alcohol (70\%, 90\%, 95\% and 100\%) 1h/each concentration. The tissues were then cleared in two changes of xylene for one hour and then infiltrated with molten paraffin wax at $60^{\circ} \mathrm{C}$ two changes for an hour. The tissues were further embedded in molten paraffin and sectioned at $5 \mu \mathrm{m}$ using a rotary microtome. The tissue sections were then stained using haemotoxylin and eosin. Light microscopic examinations were performed and presented as plates.

Statistical analysis was performed using analysis of variance (ANOVA) and Student's t-test. Experimental data were presented as mean \pm standard deviation (SD). Values of $\mathrm{p}<0.05$ were considered to be statistically significant. 


\section{Results}

\section{Effects of Artemether on the Morphology of Rat Foetuses and AFP}

The mean crown-rump length (CRL) of the foetuses in the treated groups were $4.47 \mathrm{~cm}, 3.84 \mathrm{~cm}$ and $3.66 \mathrm{~cm}$ for Groups 2, 3 and 4, respectively. The means of Groups 3 and 4 showed a significant decrease when compared to control $(4.74 \mathrm{~cm})$ at $\mathrm{p}=0.05$. The number of viable foetuses ranged between seven and ten which corresponded to the number of placental site noted. No dead foetuses and resorption sites were observed in any of the experimental groups. Significant reduction $(\mathrm{P}<0.05)$ was observed in rats in Groups 2,3 , and 4 compared to the control with respect to foetal body weight, CRL and AFP (Table 1). There was a dose-dependent reduction in the CRL of the foetuses.
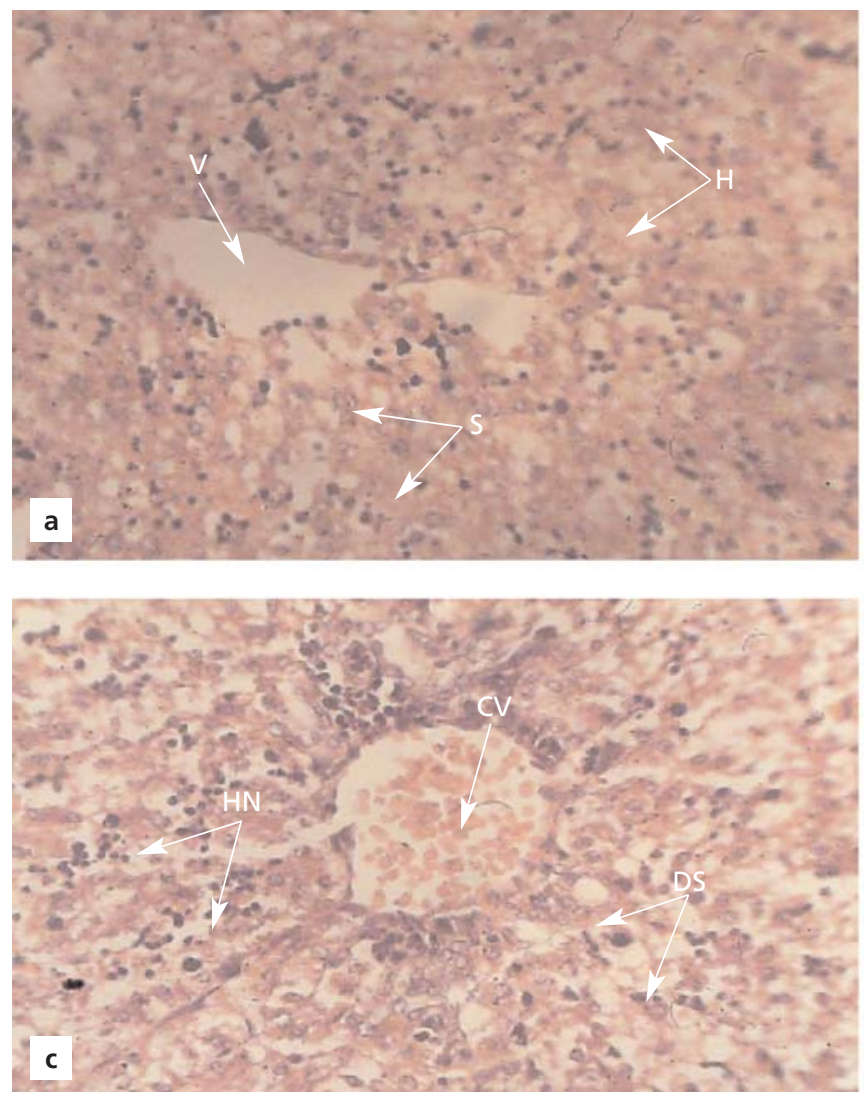

Table 1

Effect of artemether on foetal body weight, crown-rump length and alpha fetoprotein.

\begin{tabular}{lccc}
\hline Groups & Foetal weight (g) & CRL (cm) & AFP \\
\hline Group 1 (control) & $5.15 \pm 0.54$ & $4.74 \pm 0.07$ & $76.32 \pm 0.91$ \\
Group 2 (1.1 mg/kg) & $4.85 \pm 0.45^{*}$ & $4.47 \pm 0.02$ & $42.50 \pm 1.43^{*}$ \\
Group 3 (2.2 mg/kg) & $3.46 \pm 0.13^{*}$ & $3.84 \pm 0.13^{*}$ & $40.18 \pm 2.1 *$ \\
Group 4 (3.3 mg/kg) & $3.48 \pm 0.13^{*}$ & $3.66 \pm 0.07^{*}$ & $40.86 \pm 1.72^{*}$ \\
\hline
\end{tabular}

*Data presented as mean \pm standard deviation (SD) * $\mathrm{p}<0.05$. AFP: alpha-fetoprotein CRL: mean crown-rump length.

\section{Effects of Artemether on the Histology of the Liver of Rats Foetuses}

Effects of artemether on the cytoarchitecture of the foetal liver of rats are shown in Figure 1. The cytoarchitecture of liver of Group 1 was not altered. The cen-
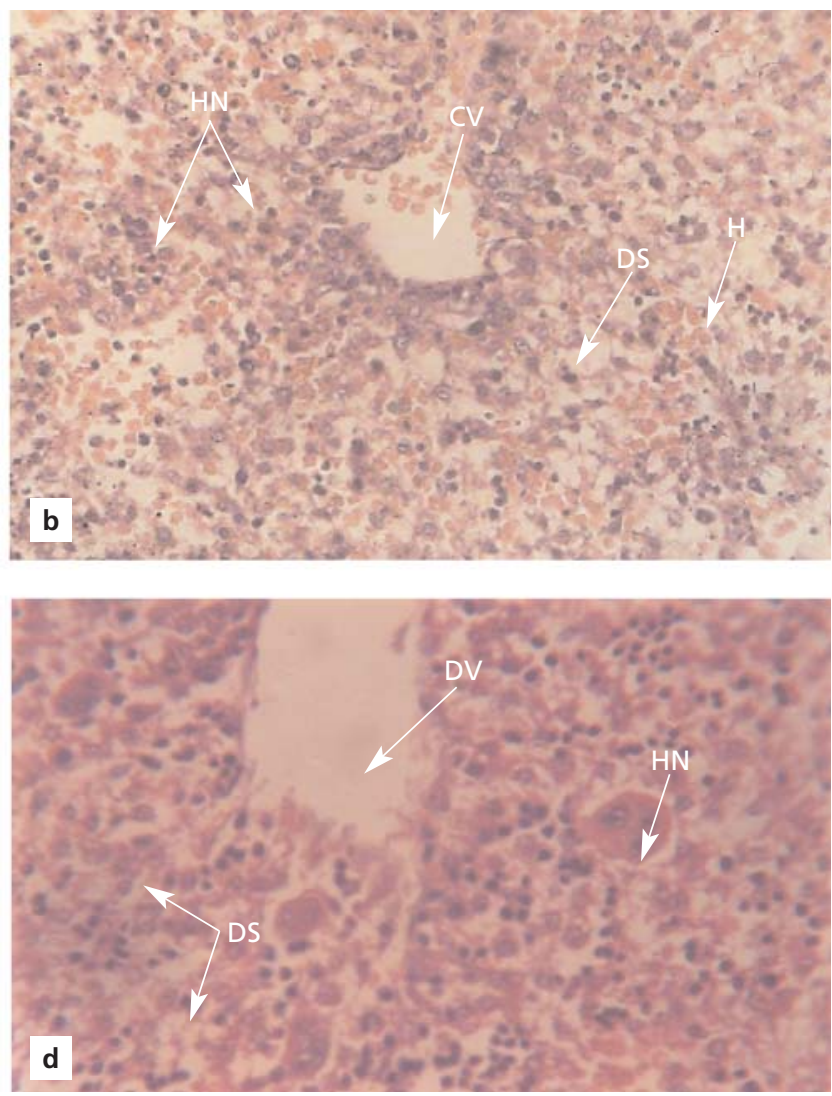

Figure 1. Foetal liver of (a) Group 1 (control group) showing normal preserved lobular architecture of the liver with normal hepatic cells, normal hepatic veins, and sinusoids. (b) Group 2 treated with $1.1 \mathrm{mg} / \mathrm{kg}$ of artemether showing moderately distorted architecture with focal hepatic necrosis, congested hepatic veins, dilated sinusoids, and haemorrhage. (c) Group 3 treated with $2.2 \mathrm{mg} / \mathrm{kg}$ of artemether showing moderately distorted architecture with focal hepatic necrosis, extensive congested hepatic veins, and dilated sinusoids. (d) Group 4 treated with 3.3 mg/kg of artemether showing severely distorted architecture with focal hepatic necrosis, severe hepatic vein dilation, dilated sinusoidal space and multinucleated giant cells. Haematoxylene and eosin stain, $\times 400$. CV: congested hepatic veins; DS: dilated sinusoids; H: normal hepatic cells; He: haemorrhage; HN: hepatic necrosis; MG: multinucleated giant cells; S: sinusoids; V: normal hepatic veins. [Color figure can be viewed in the online issue, which is available at www.anatomy.org.tr] 
tral veins and the sinusoids were prominent while the hepatocytes were well outlined (Figure 1a). The foetal liver from Group 2 animals (Figure 1b) treated with therapeutic dose $(1.1 \mathrm{mg} / \mathrm{kg})$ of artemether showed distorted architecture with marked inflammation. The central veins were dilated and congested. The sinusoids showed mononuclear infiltrate, and extensive hepatic necrosis was observed when compared to the control. There was an extensive hepatotropic necrosis with dilated sinusoidal spaces infiltrated with mononuclear inflammatory cells in the liver of Group 3 (Figure 1c), while the liver of Group 4 showed distorted liver cytoarchitecture with extensive macrovesicular steatosis and hepatic necrosis with multinucleated cells (Figure 1d).

\section{Effects of Artemether on the Histology of the Kidney of Rat Foetuses}

Effects of artemether on the cytoarchitecture of the foetal kidney of rats are shown in Figure 2. The foetal kidney of Group 1 (Figure 2a) showed distinct cortical medullary region, while moderate degeneration of glomeruli and narrowing of the capsular space were observed in Group 2 (Figure 2b). The foetal kidney of Group 3 showed disorganised renal structural details, atrophic and degenerated glomeruli with acute tubular necrosis (Figure 2c). Severe degenerated glomeruli with periarterioler haemorrhages and acute tubular necrosis were observed in foetal kidney of Group 4 (Figure 2d).

\section{Discussion}

In this study, foetal body weight and CRL were used to evaluate foetal growth morphology. The result revealed a reduction in the weight and CRL of the foetuses following administration of artemether. This is suggestive of intrauterine growth retardation. Similar observation was also made when artesunate was administered to pregnant Wistar rats in order to study its effect on the morphometry of foetal central nervous system. ${ }^{[15]}$ Observations on the foetal weight and CRL of Wistar albino rats have been reported following the administration of artesunate, artemether and arteether, ${ }^{[9]}$ Aloe vera ${ }^{[16]}$ or pyrimethamine. ${ }^{[17]}$ Ekanem et al. ${ }^{[18]}$ also reported that pyrimethamine had a deleterious effect on the foetal epiphysis leading to growth retardation and developmental anomalies.

AFP is measured in pregnant women using maternal blood or amniotic fluid as a screening test for a subset of developmental abnormalities. It is particularly increased in open neural tube defect and decreased in Down syndrome. It has also been used as a biomarker to detect tumours. ${ }^{[14]}$ There has been a correlation between amniotic fluid AFP
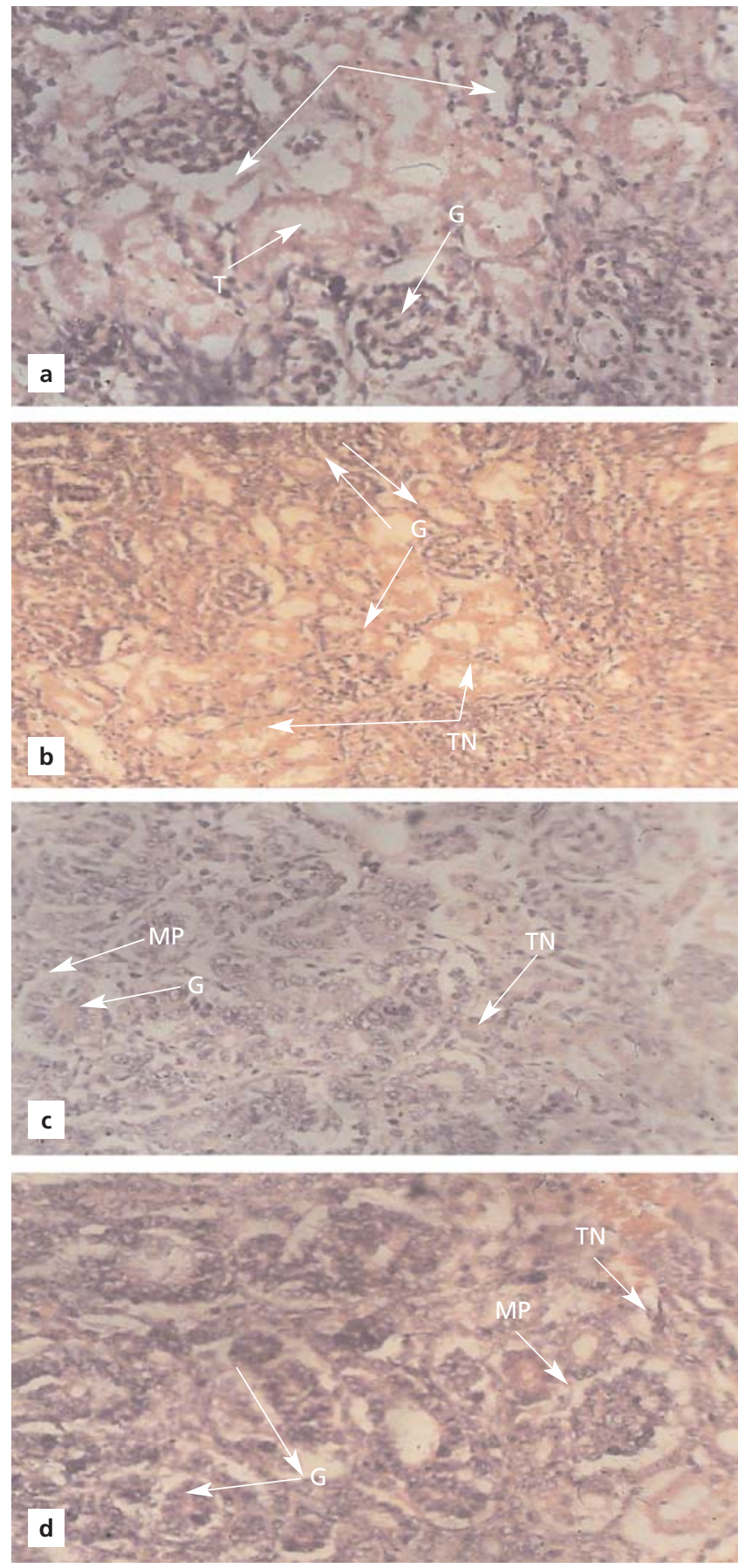

Figure 2. Foetal kidney of (a) Group 1 (control group) showing normal preserved architecture with normal glomeruli and tubules, and no tubular necrosis. (b) Group 2 rats treated with $1.1 \mathrm{mg} / \mathrm{kg}$ of artemether showing mildly distorted architecture with paucity of glomeruli, mesangial proliferation and tubular necrosis. (c) Group 3 rats treated with $2.2 \mathrm{mg} / \mathrm{kg}$ of artemether showing mildly distorted architecture with few glomeruli, mesangial proliferation and tubular necrosis. (d) Group 4 rats treated with $3.3 \mathrm{mg} / \mathrm{kg}$ of artemether showing severely distorted architecture with paucity of glomeruli, mesangial proliferation, tubular necrosis and peritubular arterioler haemorrhage. Haematoxylene and eosin stain, x400. G: glomeruli, MP: mesangial proliferation; $\mathrm{PH}$ : peritubular arterioler haemorrhage; T: tubules; TN: tubular necrosis. [Color figure can be viewed in the online issue, which is available at www.anatomy.org.tr] 
and occurrence of neural tube defects. ${ }^{[19]}$ The AFP in this study was significantly reduced in treatment groups compared to the control, implying that there was no gross foetal malformation except histologically, the liver which is another source of AFP had been significantly affected in the treated groups. This findings support the study by Bhabagrahi et al..$^{[9]}$ which reported that artemisinin has significant embryo-foetal toxicity in rats without any gross malformation. Elevation of AFP has been reported to result from the leakage of foetal serum through a disrupted and necrotic nervous tissues into the amniotic fluid. ${ }^{[20]}$

Most drugs are metabolised and eliminated in the liver and kidney, but the liver is more susceptible to drug toxicity. ${ }^{[21]}$ Teratogenic effects of drugs have been well documented ${ }^{[19]}$ Hepatocyte necrosis, hepatitis, cholestasis and fibrosis are common manifestations of liver toxicity. In this study, we observed derangements and degenerations in the foetal liver of the rats with increasing doses of artemether compared to the control. Cellular damage and a progressive cytoarchitectural damage of the foetal liver were observed in the treated animals. Congested and dilated sinusoids and central veins, hepatocytic necrosis and microvesicular steotosis were observed. Toxicological study of Ginkgo biloba in mice foetus by Zehra et al. ${ }^{[2]}$ also reported similar findings.

The kidney filters and removes toxic substances from the body. Certain drugs have been found to be nephrotoxic. Mild to severe alteration in the histology of the foetal kidney was observed in this study following the administration of artemether. The degeneration of renal glomeruli and the disorganisation of the renal structural details in this study were similar to other studies where teratogenic nephrotoxicity of Aloe vera ${ }^{[16]}$ beer and palm wine ${ }^{[23]}$ were reported.

\section{Conclusion}

The results of this study showed that artemether crosses the uteroplacental barrier to cause intrauterine growth retardation or stunted growth, as well as foetal hepatotoxicity and nephrotoxicity in Wistar albino rats.

\section{References}

1. World Health Organisation. WHO global malaria programme: World Malaria Report 2014. Geneva: WHO Press; 2014.

2. World Health Organisation. The WHO prequalification project. The global portfolio of new antimalarial medicines under development. Clin Pharmacol Ther 2009;85:584-95.

3. Burke E, Deasy J. Hasson R, McCormack R, Randhawa V, Walsh P. Antimalarial drugs from nature. Trinity Student Medical Journal 2003;4:19.
4. Mayxay M, Thongpraseuth V, Khanthavong M, Lindegardh N, Barends M, Keola S, Pongvongsa T, Phompida S, Phetsouvanh R, Stepniewska K, White NJ, Newton PN. An open, randomised comparison of artesunate plus mefloquine vs dihydroartemisininpiperaquine for the treatment of uncomplicated Plasmodium falciparum malaria in the Lao People's Democratic Republic (Laos). Trop Med Int Health 2006;11:1157-65.

5. Faye B, Ndiaye JL, Tine R, Sylla K, Gueye AH, Colle LA, Gaye OA. Randomised trial of artesunate/mefloquine vs artemether/lumefantrine for the treatment of uncomplicated Plasmodium falciparum malaria in Senegalese children. Am J Trop Med Hyg 2010;82:140-4.

6. Frey SG, Chelo D, Kinkela MN, Djouknone F, Tietche F, Hatz C, Weber P. Artesunate-mefloquine combination therapy in acute Plasmodium falciparum malaria in young children: a field study regarding neurological and neuropsychiatric safety. Malar J 2010;9: 291-9.

7. Akpanyung EO, Bassey UE, Usoh I, Iba IU. Effect of combined administration of artequin and pefloxacin on some indices of liver and renal functions of male Wistar albino rats. PharmacologyOnLine 2015;3:84-90.

8. Etim OE, Bassey UE, Charles GE, Sambo EE, Akpan EJ. Toxicological evaluation of some artemisinin combination therapies (acts) on the kidney and liver of Wistar albino rats. International Journal of Biochemistry Research and Review 2016;9:1-5.

9. Bhabagrahi R, Jyotirmoyee J, Satyajit S, Bandana R. Reproductive profile of artemisinins in albino rats. Indian J Pharmacol 2010;42: 192-3.

10. El-Dakdoky MH. Evaluation of the developmental toxicity of artemether during different phases of rat pregnancy. Food Chem Toxicol 2009; 47:1437-41.

11. Li Q, Weina PJ. Severe embryotoxicity of artemisinin derivatives in experimental animals, but possibly safe in pregnant women. Molecules 2009;15:40-57.

12. Clarke RL, Lerman SA, Cox EM, Gristwood WE, White TE. Developmental toxicity of artesunate in the rat: comparison to other artemisinins, comparison of embryotoxicity and kinetics by oral and intravenous routes, and relationship to maternal reticulocyte count. Birth Defects Res (Part B) 2008;83:397-406.

13. Longo M, Zanoncelli S, Torre PD, Riflettuto M, Cocco F. Effects of the antimalarial drug dihydroartemisinin (DHA) on rat embryos in vitro. Reprod Toxicol 2006;21:83-93.

14. Harpey JP, Darbois Y, Lefebvre G. Teratogenicity of pyrimethamine. Lancet 1983:2:399.

15. Mesembe OE, Inyang AE, Udoaffah G, Igiri AO, Fischer VA, Akpaso M, Eluwa MA. Akpa OA. A morphometric study of the teratogenic effect of artesunate on the central nervous system of the Wistar rat fetuses. Nigerian Journal of Physiological Sciences 2004; 19:1-12.

16. Eluwa MA, Otung GO, UDo-afah G, Ekanem TB, Mesembe OE, Aligweke AU. Teratogenic effect of maternal administration of aloe vera extract on fetal morphology and the histology of the fetal kidney. Global Journal of Medical Sciences 2006;5:41-4.

17. Akpan TB, Ekanemesang UM, Ebong PE, Singh ED. Teratogenic induction of skeletal anomalies by pyrimetamine (Daraprin) in Wistar rats fetuses (Rattus Norvegicus): a morphological study. West African Journal of Anatomy 1992;1:90-3. 
18. Ekanem TB, Ekanemesang UM, Ekanem P. Teratogenic effect of pyrimethamine in epiphysial rats fetuses. West African Journal of Anatomy 1999;6:9-12.

19. Moore KL, Persuad TV. The Developing human. Clinically oriented embryology. 7th ed. Philadelphia, PA: W. B. Saunders; 2003. p. 547-62.

20. Peter PW, Verhoef A, Hagenaars AM. Amniotic and maternal serum alpha fetoprotein of rats with induced neural tube defect. Scandinavian Acta of Morphology 1981;19:205-16.

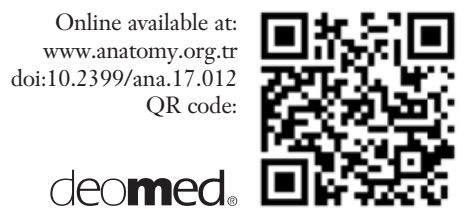

21. Abd-Elmagid BF, Al-Gharudiz FB. Effect of administration of haloperidol on the developing fetus of the chick embryo. Saudi J Biol Sci 2005;15:297-306.

22. Zehra U, Tahir M, Jafferry FH, Lone KP. Ginkgo biloba effects of mice fetal liver. Int J Morphol 2010;28:765-70.

23. Eluwa M, Ekere E, Ekanem T, Akpantah A, Igiri A. Teratogenic effect of beer and palmwine on the histology of the fetal kidney of Wistar rats. The Internet Journal of Toxicology 2009;6:1-5.

Correspondence to: Idorenyin Udo Umoh, MD Department of Anatomy, Faculty of Basic Medical Sciences, University of Uyo, Uyo, Akwa Ibom State, Nigeria Phone: +2348036743886

e-mail: umohidorenyin_dr@yahoo.com

Conflict of interest statement: No conflicts declared.

This is an open access article distributed under the terms of the Creative Commons Attribution-NonCommercial-NoDerivs 3.0 Unported (CC BY-NCND3.0) Licence (http://creativecommons.org/licenses/by-nc-nd/3.0/) which permits unrestricted noncommercial use, distribution, and reproduction in any medium, provided the original work is properly cited. Please cite this article as: Umoh IU, Jimmy EO. Foetal hepatorenal toxicity and intrauterine growth retardation in pregnant Wistar rats treated with artemether. Anatomy 2018;12(1):1-6. 